\title{
DIRECT FARADAY ROTATION ANGLE RETRIEVAL IN SMOS FIELD OF VIEW
}

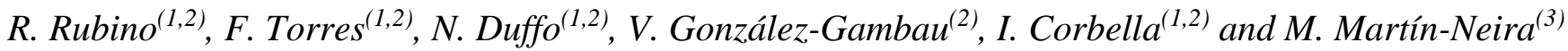 \\ ${ }^{(1)}$ Remote Sensing Laboratory, Universitat Politècnica de Catalunya, Barcelona, (SP) \\ ${ }^{(2)}$ Barcelona Expert Centre. Institute of Marine Sciences, CSIC. xtorres@tsc.upc.edu \\ (3) European Space Agency (ESA)
}

\begin{abstract}
It has recently been demonstrated that boresight averaged Faraday rotation angle (FRA) can be retrieved directly from SMOS full-pol radiometric data. However, in order to extend FRA retrievals to the full Alias-Free Field of View (AF-FoV), SMOS relatively poor pixel radiometric sensitivity and accuracy must be compensated by spatial and temporal averaging. This requires some kind of tradeoff to constrain systematic FRA estimation bias both within SMOS AF-FoV and along the orbit. This work presents the first results given by a SMOS end-to-end FRA simulator, currently under development, that is used to trim and assess the performance of several FRA retrieval approaches.
\end{abstract}

Index Terms - Faraday rotation, L-band radiometry, Interferometric synthetic aperture radiometry, SMOS.

\section{INTRODUCTION}

SMOS stands for the global Soil Moisture and Ocean Salinity mission by the European Space Agency (ESA) [1]. Its single payload, MIRAS (Microwave Imaging Radiometer with Aperture Synthesis) [2] is responsible for measuring the full polarimetric brightness temperature of the Earth surface, used in scientific models for estimating both geophysical parameters. Since Faraday rotation is not negligible at SMOS operating frequency $(1.41 \mathrm{GHz})$, it must be compensated for before geophysical parameter retrieval is undertaken from measured polarimetric brightness temperature maps. FRA is currently estimated indirectly by using a formulation that makes use of data provided by external sources to get the necessary TEC and geomagnetic field [3].

Improved geophysical parameter retrievals require getting the Faraday rotation directly from measured full-polarimetric radiometric data [4]. Recent improvements in the image reconstruction algorithms, as the so-called, full-pol G-matrix, used in the last release of SMOS L1 operational processor, provides improved third and fourth stokes parameters [5].

\section{FARADAY ROTATION BASICS}

The electromagnetic field components of microwave radiation from earth rotate an angle, called Faraday Rotation
Angle (FRA), as they propagate through the ionosphere. The magnitude of FRA depends on the total ionospheric electron content (TEC), the frequency, the geomagnetic field and observation geometrical configuration. In SMOS current data processing all these parameters are known from external sources and from the instrument configuration and attitude. Particularly, the so-called "consolidated TEC" is available globally with a delay typically of 15 days since radiometric data acquisition. For faster processing, an estimation of the TEC is also available, although with less quality. Using these data, Faraday rotation angle can be computed (in degrees) using [6]:

$$
\Omega_{f}=1.355 \times 10^{4} f^{-2} B_{0} \cos \Theta_{B} \sec \theta \text { VTEC }
$$

with $\boldsymbol{f}$ the frequency in $\mathrm{GHz}, B_{0}$ the geomagnetic field at 400 $\mathrm{km}$ height in Tesla, $\Theta_{B}$ the angle between the magnetic field and the wave propagation direction, $\theta$ the angle between the wave propagation direction and the vertical to the surface and VTEC the vertical total electron content in TEC units $\left(10^{16}\right.$ electrons $/ \mathrm{m}^{2}$ ).

The rotation matrix of the polarimetric brightness temperature from ground to antenna frame depends on the total rotation angle, which is the sum of the geometrical (Ludwig) and the Faraday contributions. If the third and fourth Stokes parameters are assumed to have negligible values compared to horizontal and vertical brightness temperature, the FRA can be computed by writing equation (22) of [6] as

$$
\Omega_{f}=-\varphi-\frac{1}{2} \arctan \left(\frac{2 \Re e\left(T_{B}^{x y}\right)}{T_{B}^{x x}-T_{B}^{y y}}\right),
$$

where $\varphi$ is the geometrical rotation angle and $T_{B}^{x y}, T_{B}^{x x}$ and $T_{B}^{x y}$ SMOS level 1 products. This direct FRA measurement can be compared to the one computed in (1) for consistency and validation procedures.

\section{FRA RETRIEVAL SIMULATOR}

Estimating FRA out of SMOS radiometric data is not straightforward due to MIRAS relatively poor accuracy (spatial bias) and radiometric sensitivity (thermal noise). To cope with these issues, FRA can be retrieved with good accuracy by averaging all pixels within a circle of radius 
$\mathrm{r}=0,3$ in the direction cosine domain [7]. Although these results have demonstrated the capability of SMOS full-pol radiometric brightness temperatures to allow direct FRA retrievals under different imaging conditions (land, ocean, RFI, etc.), improved operational geophysical parameter retrievals require accurate FRA correction for all pixels within the FoV.

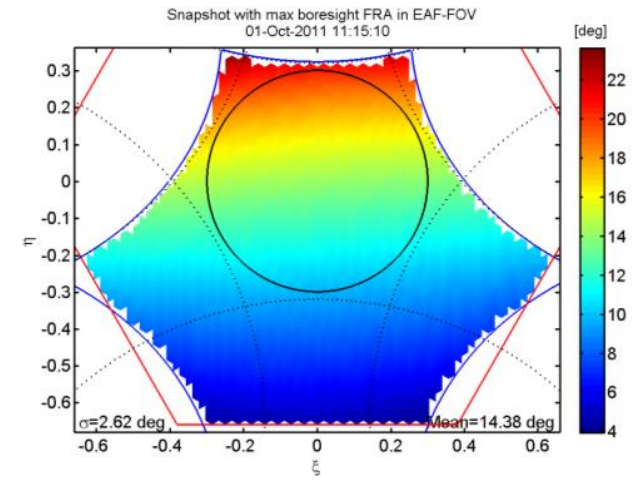

Fig. 1. FRA distribution in SMOS Alias-Free Field of View (AFFoV) for a snap-shot in a descending orbit over Indian Ocean. The circle $r=0,3$ is used in [7] to give a boresight averaged FRA.

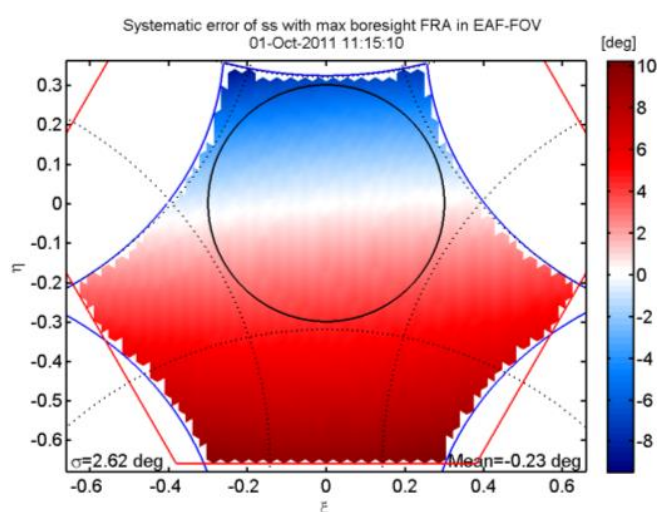

Fig. 2. FRA systematic bias if boresight averaged FRA is assigned to all pixels in SMOS AF-FoV (worst case).

In order to assess the performance of several FRA retrieval approaches, a FRA end-to-end simulator is currently under development. The forward section makes use of a simple geophysical model of the Earth. Ground emissions are simulated by means of a Fresnel model of the Ocean (35 psu at $21 \mathrm{C}$ ) and land is set to a constant brightness temperature $(280 \mathrm{~K})$. FRA in any direction is then computed out of SMOS auxiliary data in order to simulate polarimetric brightness temperature images at SMOS antenna frame. Once the test images are available, simulated SMOS polarimetric visibility samples are computed by means of the G-matrix. Finally, a G-matrix Moore-Penrose pseudoinverse is used to produce simulated SMOS full-pol polarimetric brightness temperature maps. These noise free images are used to estimate FRA retrieval systematic bias when temporal or spatial averaging is applied. In particular, they are used to assess the regions where FRA shows large geographical and temporal variations and averaged boresight FRA estimations are not representative across the full SMOS AF-FoV. Fig.1 shows a typical FRA map in a descending orbit over the Indian Ocean. In this case, as shown in Fig. 2, if the averaged boresight FRA $\left(14^{\circ}\right.$ in the circle $\left.\mathrm{r}=0,3\right)$ is assigned to all pixels in the AFFoV a large systematic bias appears across the AF-FoV.

When radiometric noise is added to the test images, the impact of spatial and temporal averaging can be assessed as a tradeoff between FRA resolution and systematic bias.

\section{CONCLUSION}

This paper presents the first results given by an end-to-end SMOS direct FRA retrieval simulator, currently under development. The simulator is used to trim and assess the performance of several direct FRA retrieval approaches.

\section{ACKNOWLEDGMENT}

This work has been supported by the European Space Agency and Deimos Engenharia (Portugal), SMOS P7 Subcontract DME CP12 no. 2015-005; ERDF (European Regional Development Fund) and by Spanish public funds, projects TEC2014-58582-R and ESP2015-67549-C3-1-R.

\section{REFERENCES}

[1] H. Barré, B. Duesmann, and Y. Kerr, "SMOS: The mission and the system," IEEE Transactions on Geoscience and Remote Sensing, vol. 46, no. 3, pp. 587-593, March 2008.

[2] K. McMullan, M. Brown, M. Martín-Neira, W. Rits, S. Ekholm, J. Marti, and J. Lemanzyk, "SMOS: The payload," IEEE Transactions on Geoscience and Remote Sensing, vol. 46, no. 3, pp. 594-605, March 2008.

[3] D. M. Le Vine and S. Abraham, "The effect of the ionosphere on remote sensing of sea surface salinity from space: Absorption and emission at L band," IEEE Transactions on Geoscience and Remote Sensing, vol. 40, no. 4, pp. 771-782, April 2002.

[4] M. Martín-Neira, S. Ribó, and A. J. Martín-Polegre, "Polarimetric mode of MIRAS," IEEE Transactions on Geoscience and Remote Sensing, vol. 40, no. 8, pp. 1755 -1768, August 2002.

[5] L. Wu, F. Torres, I. Corbella, N. Duffo, I. Durán, M. Vallllossera, A. Camps, S. Delwart, and M. Martín-Neira, "Radiometric performance of SMOS full polarimetric imaging," IEEE Geoscience and Remote Sensing Letters, vol. 10, no. 6, pp. 1454-1458, November 2013.

[6] S. H. Yueh, "Estimates of Faraday rotation with passive Microwave polarimetry for microwave remote sensing of earth surfaces," IEEE Transactions on Geoscience and Remote Sensing, vol. 38, no. 5, pp. 2434-2438, September 2000.

[7] I. Corbella, L. Wu, F. Torres, N. Duffo and M. Martin-Neira. "Faraday Rotation Retrieval Using SMOS Radiometric Data". IEEE Geoscience \& Remote Sensing Letters, Vol.12, iss. 3, pp. 458461. 2015. 